\title{
Gait Termination on Declined Compared to Level Surface; Contribution of Terminating and Trailing Limb Work in Arresting Centre of Mass Velocity
}

\author{
Zahraa M Abdulhasan ${ }^{\mathrm{a}}$, John G Buckley ${ }^{\mathrm{a},{ }^{,}}$
}

${ }^{a}$ Department of Biomedical and Electronics Engineering, University of Bradford, BD7 1DP, UK

*corresponding author: email, j.buckley@bradford.ac.uk 


\section{Abstract}

1 To terminate gait, the mechanical work-done by the lower-limbs is likely to be

2 predominantly negative but how such work is produced/completed has not previously

3 been investigated. The aim of this study was to determine the amount of negative

4 mechanical (external) work-done by the lower-limbs, along with the associated joints

5 (muscle) work, to terminate gait and how these work contributions were affected by a

6 change in surface angle.

7 Eight males completed terminations on the level floor and a declined ramp. Negative

8 mechanical limb-work (limbW $($-ve) $)$ was computed (each orthogonal direction) as the

9 dot-product of the ground-reaction-force and centre-of-mass (CoM) velocity. Inverse dynamics was used to calculate ankle, knee and hip negative joints (muscle) work $\left(\mathrm{Wj}_{\text {(-ve) }}\right)$. Measures were determined for each limb for the two-locomotor steps of gait termination.

The trailing-limb did $67 \%(-0.386 \mathrm{~J} / \mathrm{kg})$ of the overall $\operatorname{limbW}_{(-\mathrm{ve})}$ to terminate gait on the level; and this increased to $74 \%(-0.451 \mathrm{~J} / \mathrm{kg})$ for ramp trials. $\mathrm{Wj}_{\text {(-ve) }}$ was greater for the trailing- (ankle -0.315 ; knee -0.357 ; hip $-0.054 \mathrm{~J} / \mathrm{kg}$ ) compared to terminatinglimb (ankle, -0.063; knee -0.051; hip -0.014 J/kg), with the increases in ankle $\mathrm{Wj}_{\text {(-ve) }}$ being temporally associated with increases in perpendicular $\operatorname{limbW}_{(-\mathrm{ve})} . \mathrm{Wj}_{\text {(-ve) }}$ increased on both limbs for declined compared to level surface, particularly at the knee (declined -0.357 , level $-0.096 \mathrm{~J} / \mathrm{kg}$ ), with such increases being temporally associated with increases in parallel $\operatorname{limbW}_{(-\mathrm{ve})}$. These findings provide new perspectives on how the limbs do work on the CoM to terminate gait, and may be helpful in designing prosthetic limbs to facilitate walking on ramps.

Keywords: Gait termination; external work; limb work; centre of mass velocity; ramp descent. 


\section{Introduction}

Ramped walkways are used as access ways to almost every public building and provide an option to avoid steps and stairs. Sloped surfaces can be considered as environmental hazards that challenge locomotor behavior[1], and walking down ramps in particular is known to increase the risk of falling compared to that for walking on level surfaces[2]. Research undertaken to determine how gait is altered when walking down a 5-deg ramp, has shown that peak normal ground reaction forces (GRF) increase by $11 \%$ and peak horizontal GRF by $66 \%$ compared to walking on a level surface[3]. Ultimately, the GRF generated reflect the mechanical work done on the whole-body centre of mass (CoM) by the lower-limbs (so called external mechanical work), and is computed as the dot product of the GRF and the instantaneous velocity of the CoM[4].

When walking on a level horizontal surface the limb initially performs negative external mechanical work on the CoM following foot contact to arrest the downward trajectory of the CoM and redirect it upwards[4]. The contralateral (trailing) limb concurrently performs positive mechanical work in order to help transfer the CoM on to the leading limb[4]. In comparison to level walking, more negative mechanical work (power absorption) is done by the limbs when walking down ramps[5], and surprisingly even during the double support phase when the CoM is being transferred from the trailing to the terminating-limb, the trailing-limb performs predominantly negative mechanical work [6]. There are no previous studies that have investigated the external mechanical work required to terminate gait and if and how such work is altered when terminating gait on a declined surface.

When terminating gait, the mechanical work done is likely to be predominantly negative in order to fully arrest (halt) CoM velocity. When descending a ramp, arresting CoM velocity must be undertaken whilst also lowering it down the ramp, and thus stopping on a declined surface will likely require even greater negative mechanical work compared to that when terminating gait on a level surface. The present study explores these suppositions. The specific aims were to determine the amount of negative external mechanical work performed by each of the lower-limbs to terminate gait and determine how such work is affected by a change in surface 
angle from level to declined. In satisfying this aim we compute the negative external mechanical work done by each limb in both the parallel, perpendicular and mediolateral directions, along with the overall negative work done by each limb. Previous research has shown that terminating gait is accomplished over two walking steps[7-10]. Based the research mentioned above, we hypothesised that during the two steps of gait termination, the limb that initiates the final step (terminating limb) will perform more negative work (power absorption) compared to that done by the contralateral trailing-limb, and that this increased work will be mostly done in the parallel direction due to having to arrest CoM forward velocity. We also hypothesised that the negative work done by the terminating-limb will increase further when terminating gait on a declined surface, and this additional increase in work will be mostly done in the perpendicular direction due to having to lower the CoM down the ramp whilst also arresting CoM forwards velocity.

To provide insight into how the lower-limbs generate the external mechanical work done in halting gait, a secondary aim of the present study was to determine the negative joints (muscle) work contributions for both the trailing- and terminating-limbs when terminating gait and determine how such joint work is affected by a change in surface angle from level to declined.

\section{Methods}

\section{Participants}

Eight healthy males (mean (SD), age27.5 (6.93) years, height $1.77(0.067) \mathrm{m}$, mass $73.54(10.74) \mathrm{kg}$ ) with no self-reported balance or gait abnormalities participated in the study, all giving written informed consent. The tenets of the Declaration of Helsinki were observed, and ethical approval was obtained from the institutional ethics committee.

\section{The ramp}

The inclined walkway was modular in design and consisted of one level section, and three angled sections that where 'bolted' together to provide a $4 \mathrm{~m}$ long by $1 \mathrm{~m}$ wide 5-degree declined walkway with a $1 \mathrm{~m}$ long level surface at its top end. The two sections at the lower end of the inclined walkway contained two solid (chip-board) 
sloped blocks which were (bolted) onto two adjacent force platforms. There was a 3$\mathrm{mm}$ gap between each side of the sloped blocks and the surrounding ramp, and the block surfaces were flush with the surface of the surrounding ramp.

\section{Experimental protocol}

Participants completed gait terminations in two blocks: with block order counterbalanced across participants. In one block, gait terminations were performed on the declined ramp and in the other they were completed over the laboratory floor. Each block included 10 repetitions. Starting either on the level section at the top end of the ramp or on the level floor, participants were asked to walk (down the ramp, over level floor) at their self-selected customary speed, and to terminate gait on the limb they indicated was their preferred (i.e. the limb they would use to kick a ball), which for all participants was their right limb. Gait terminations occurred 5 to 6 walking steps from the starting location, which was adjusted for each participant so that gait terminations occurred with the final two steps landing consecutively within the bounds of the two adjacent force-platforms or the sloped blocks above the platforms, i.e. left foot landing within bounds of platform 2, right foot landing within bounds of platform 1 .

\section{Data acquisition and processing}

Segmental kinematics and GRF data were collected $(200 \mathrm{~Hz})$ using a 10-camera motion capture system (Vicon MX, Oxford, UK) incorporating two strain-gauge force plates $\left(508^{*} 464 \mathrm{~mm}, \mathrm{AMTI}\right.$, Watertown, MA, USA). Retro-reflective markers were placed on the following locations (Figure 1): bilaterally on iliac crest (vertically above each trochanter when standing), greater trochanter, medial and lateral femoral condyles, medial and lateral malleoli, posterior aspect of calcaneus, superior aspects of first and fifth metatarsal heads, distal end of second toe, and pragmatically on the lateral and medial aspects of the proximal and distal mid-foot. In addition, 4-marker clusters (semi-rigid plates with four non-collinear markers) were placed on the lateral aspect of thighs and shanks, and over the sacrum. For the upper body, markers were placed on acromion processes, sternal notch, xiphoid process, and vertebrae $\mathrm{C} 7$ and T8. A headband was used to mount 4 head markers (left/right temples, left/right back of the head). Following collection of a standing calibration trial and 
trials in which the limbs were 'waggled' in order to determine functional joint centres (see below), the markers on the medial femoral condyles, medial malleoli and the acromion processes were removed. Labelling and gap filling were done using Vicon Nexus 1.8.5 software. Data were subsequently exported in C3D format to Visual3D software (Version 5.02.27 C-Motion, Germantown, MD, USA) where all further processing took place.

A six degrees of freedom nine segment model [11] was created for each participant. Joint centres were determined using a functional joint centre approach using data from the limb 'waggling' trials [12]. The location of the whole-body centre of mass (CoM) was determined as the weighted average of the nine tracked segments [13]. Data were filtered using a fourth order, zero-lag Butterworth filter with $6 \mathrm{~Hz}$ and $20 \mathrm{~Hz}$ cut-off for marker coordinate and GRF data respectively. For ramp trials, a force structure representing the dimensions and location of each sloped surface was created above each force platform. This allowed the centre of pressure coordinates to be transformed (within Visual 3D) from each platform surface to the top surfaces of each 'force structure'.

\section{Data analysis}

Foot contact (trailing/left limb, terminating/right limb) and toe-off (trailing-limb only) events were defined as the instants the vertical GRF force first went above or below $50 \mathrm{~N}$ respectively. Instant of final bipedal standing (on platform 1) was defined as the instant the CoP medial velocity under the terminating-limb first went above $0.2 \mathrm{~m} / \mathrm{s}$ following toe-off of trailing-limb (from platform 2): pilot work indicated that this coincided with the beginning of limb-loading/contact of the trailing limb on platform 1 (i.e. as the trailing limb is loaded the CoP rapidly moves medially from the terminating-limb towards the trailing-limb).

The following parameters were determined:

Walking speed: CoM forwards (antero-posterior) velocity at instant of trailing-limb foot contact (on platform 2). 
160 Braking-phase $\left(\right.$ Brk $\left._{\mathrm{T}}\right)$ duration: time period from foot contact of each limb up to contralateral-limb foot contact.

Limb directional-power: dot product of GRF under each limb and instantaneous velocity of the CoM; determined separately for the directions parallel, perpendicular and mediolateral to the walking surface [4][6]:

$$
\begin{aligned}
& P_{\text {perp }}=F_{z} \cdot V C o M_{\text {perp }} \text { (1) } \\
& P_{p a r}=F_{y} \cdot V C o M_{p a r} \text { (2) } \\
& P_{M L}=F_{x} \cdot V \operatorname{VOM}_{M L} \text { (3) }
\end{aligned}
$$

Where Fx, Fy, and Fz are the GRF (normalised to body mass) under each limb in the side-to-side, parallel (A-P direction), and perpendicular directions respectively, and VCoM (ML/perp/par) are the CoM instantaneous velocities in the side-to-side (M-L), perpendicular (perp) and parallel (par) directions respectively.

Limb total power: summation of parallel, perpendicular and mediolateral power:

$$
P_{\text {tot }}=P_{\text {perp }}+P_{p a r}+P_{M L} \text { (4) }
$$

Limb negative directional-work: time integral of negative power in each orthogonal direction, restricted to braking phase of each limb:

$$
\begin{aligned}
\operatorname{Limb} W_{\text {perp (-ve) }} & ={ }_{B r k T} \int P_{\text {perp }} d t(5) \\
\operatorname{Limb} W_{p a r}(-v e) & ={ }_{B r k T} \int P_{\text {par }} d t(6) \\
\operatorname{Limb} W_{M L}(-v e) & ={ }_{B r k T} \int P_{M L} d t(7)
\end{aligned}
$$

Limb negative total work: time integral of negative total power, restricted to braking phase of each limb:

$$
\text { Limb } W_{\text {tot }}(- \text { ve })={ }_{\text {Brk }} \int P_{\text {tot }} d t(8)
$$

Joint [muscle] power: dot product of joint sagittal moment (M, normalized to body mass) and angular velocity of joint ( $\omega)$ [14]:

$$
P_{j}=M . \omega(9)
$$


Joint negative work: integral of negative joint power, restricted to braking phase for each limb:

$$
W_{j(-v e)}={ }_{B r k T} \int P_{j} d t(10)
$$

Outcomes variables were determined for each limb for each trial and then averaged across trials to give mean values for each surface condition (level, ramp) per participant.

\section{Statistical analysis}

Limb directional- and limb total- negative work were compared using repeated measures analysis of variance (ANOVA) with surface condition (level, ramp) and limb (trailing, terminating) as repeated factors. Joint negative work was compared using repeated measures ANOVA with surface condition (level, ramp), limb (trailing, terminating), and joint (ankle, knee, hip) as repeated factors. Post-hoc analyses were undertaken using Tukey HSD tests. Statistical analyses were performed using Statistica (StatSoft, Inc., Tulsa, OK, USA). The alpha level was set at 0.05 .

As highlighted above, our analysis focussed on comparing mean outcome variables (calculated across the 10 repetitions) between surface conditions and between limbs. To confirm that we were justified in assessing mean outcome variables (rather than for example, comparing the minimum or maximum), we used preliminary statistical analysis to assess if there were any differences across repetitions (e.g. learning and/or trial/fatigue effects). For this analysis we included repetition as a repeated measures factor within the ANOVA model. Although this analysis indicated there was a main effect of repetition for the amount of negative limb work done ( $p$ > 0.03) post-hoc analysis indicated there were no significant differences between repetitions $(p>0.23)$. In addition, there were no repetition by limb or repetition by surface interaction effects $(p>0.53)$.

\section{Results}

Group ensemble average directional- and total- power profiles for each limb for ramp and level trials are presented in Figure 2. Group mean ( $\pm S D$ ) directional- and totalnegative work values for each limb for ramp and level trials are depicted in Figure 3. 
225 Group ensemble average joint (muscle) power profiles for the ankle, knee and hip for each limb for ramp and level trials are presented in Figure 4.Group mean negative joint (muscle) work for the ankle, knee and hip for each limb are presented in Figure 5.

Group average walking speed was $1.14(0.16)$ and $1.08(0.27) \mathrm{m} / \mathrm{s}$ for level and declined surface respectively $(p=0.69)$.

Limb directional negative work in all three orthogonal directions was significantly affected by limb $(\mathrm{p}<0.03)$. More negative work was done by the trailing-compared to terminating-limb in the perpendicular and parallel-AP directions but in the ML direction less work was done by the trailing-compared to terminating-limb. Limb perpendicular negative work was also significantly affected by surface $(p=0.0004)$ and by a limb by surface interaction $(p=0.025)$ : but there were no differences between surface conditions or limb by surface interactions in the other two directions $(P>0.37)$. More limb perpendicular negative work was done for declined compared to level surface, and the limb-by-surface interaction indicated this increase was mainly due to more work being done on the declined surface by the trailing-limb $(p=0.006)$ with little difference between surface conditions for the terminating-limb $(p=0.77)$.

Limb negative total work was significantly affected by limb $(p<0.001)$ but not by surface $(p=0.34)$ : the interaction between terms approached significance $(p=0.055)$. Limb negative total work was greater for the trailing-limb $(-0.386,-0.451 \mathrm{~W}$ for level and decline surface respectively) compared to terminating-limb $(-0.193,-0.160 \mathrm{~W}$ for level and decline surface respectively). The trend interaction between limb and surface indicated that trailing-limb negative work slightly increased on declined surface whilst the contribution of the terminating-limb decreased slightly; however post-hoc analyses indicated differences were non-significant.

Joint negative work was significantly affected by limb, by surface and by joint $(p<0.001)$. There was also limb by surface, limb by joint and surface by joint $(p<0.001)$ interactions, and a significant three-way interaction $(p<0.001)$. Joint negative work was greater for the trailing-compared to terminating-limb, and 
increased on both limbs for declined compared to level surface but post-hoc analyses of the limb-by-surface interaction indicated only increases for the trailinglimb were significant $(p<0.001)$. More work was done at the ankle compared to knee which in turn was greater than work done at the hip; however, post-hoc analyses of the limb-by-joint interaction indicated differences between joints were only significant for the trailing-limb $(p<0.001)$. The surface-by-joint interaction indicated the increased negative joint work done on declined surface was mainly due to increased knee work $(p<0.001)$ with negligible increases at the ankle or hip $(p>0.46)$. Post-hoc analysis of the three-way interaction did not reveal anything different to what the three 2-way interactions (detailed above) indicated.

\section{Discussion}

The present study determined the amount of external negative mechanical work done by each limb to terminate gait and how such work was affected by a change in surface angle from level to declined. Counter to what was hypothesised, results indicate that the mechanical work done to halt gait was done mainly by the trailinglimb irrespective of surface angle. Specifically, the trailing-limb did $67 \%$ of the overall negative work undertaken by both limbs to terminate gait on the level; and this increased to $74 \%$ in ramp trials. This means the limb that gait was terminated on only did $33 \%$ and $26 \%$ of the overall negative work in level and ramp trials respectively. The greater negative mechanical work done by the trailing-limb, was due to higher magnitude negative limb power in the parallel direction throughout the braking phase, and to a period of negative limb power in the perpendicular direction during the latter part of the braking phase (Figure 2 and Figure 3). The higher magnitude negative limb power in the parallel direction throughout the braking phase reflects the increased negative mechanical work done by the trailing-limb to arrest the CoM velocity. The increased negative limb power in the perpendicular direction for ramp compared to level trials would have been a result of the increased lowering of the CoM in such trails. There was no negative limb power in the perpendicular direction evident for the terminating-limb, apart from a very brief during period immediately following foot contact. This would be expected as the CoM was halted on this limb rather than being lowered/transferred to the next step. The mechanical limb total power profile for the trailing-limb for terminations on both level and decline 
291 is similar (in shape and magnitude) to that reported for constant speed walking on a decline[6].

Previous research has shown that the terminating-limb contributes considerably more of the braking (A/P GRF) force compared to the trailing-limb when terminating gait on the level[10,15]. In the present study A/P GRFs were likewise greater for the terminating compared to trailing-limb for terminations on both the level and decline. During planned/predicted stopping there is advanced information available regarding the distance and conditions of the future stopping location which can be used to determine the most efficient stopping strategy[9-10,16]. In such 'predicted' gait terminations the CoM velocity undergoes preparatory braking during the first step when it losses around $10 \%$ of forward speed before it undergoes rapid braking during the final step[8]. As calculation of the external limb work takes in to account not only the magnitude of the GRFs but also the magnitude of the instantaneous CoM velocity, a rapidly reducing CoM velocity during the final step would result in considerably reduced external limb work. This explains why in the present study the limb work done by the terminating-limb ('last step') was considerably less than that done by the trailing-limb ('second-last step') even though the braking forces were greater for the terminating-compared to trailing-limb.

The greater negative mechanical work done by the trailing- compared to terminatinglimb was associated with greater amounts of negative joints work on the trailing-limb, particularly at the ankle during the latter part of the braking phase (Figure 4 and 5). Negative ankle joint work done in the latter part of the braking phase reflects the control exerted on the shank to govern how quickly it rotated forwards over the planted foot during single-limb support, which in turn governed how quickly the CoM progressed over the planted foot. At the onset of trailing-limb single-support the CoM would be forward of the ankle and thus would begin to 'fall' (inverted pendulum). As highlighted above, negative limb power in the perpendicular direction also increased during the latter part of single-limb support (Figure 2). This suggests that the limb negative power in the perpendicular direction and the negative ankle joint power 
control the lowering of the CoM during single-limb support (i.e. acts to control inverted pendulum).

For gait terminations on the declined surface, knee negative joint work markedly increased $(p=0.0005)$, particularly on the trailing-limb. This suggests that kinetic adaptations at the knee are important in controlling the increased CoM lowering required for ramp descent. This finding is in agreement with studies showing that during downslope walking, peak power absorption increases markedly at the knee joint compared to that for when walking on the level[17, 5]. In the present study, it is worth noting that the increase in trailing-limb negative knee joint work for ramp compared to level trials was due to increased knee negative power during both the initial and final part of stance (Figure 4). The increase in knee negative joint power during initial stance corresponds (temporally associated) with a period of negative limb power in the parallel direction (Figure 2). This suggests that negative knee joint work is predominant in reducing CoM forwards velocity during limb loading (weight acceptance period), particularly so for gait terminations on a ramp; as evidenced by the increased negative knee joint power during this period compared to level trials. The period of increased knee negative joint power during late stance 'peaked' in magnitude after contralateral limb contact, and appeared not to be temporally associated with any directional component of limb power. This suggests that the knee was flexing compliantly (no effect on the CoM) during late stance. Such compliant flexion may have occurred to ensure there was a minimal increase in CoM height, as it was transferred from the trailing- to the terminating-limb.

Previous research has shown that during level walking the total limb power (combined parallel, perpendicular and mediolateral) has a negative followed by positive phase[4], and when walking downslope, the total limb positive work reduces, and the negative work increases (and during upslope walking the total limb positive work increases while the negative work reduces)[6]. In the present study, although the total limb power for both limbs was predominantly negative, there was a period of positive total limb power on both limbs (though such was negligible for the terminating-limb in levels trials) during the early-to-mid part of the braking phase. Previous research has indicated that positive limb power during this period in stance 
(i.e. double-support period) is due to the 'push' from the contralateral limb, i.e.

transition from one inverted pendulum (limb) to the next [4]. As evident in the present study this 'push' was a result of positive limb power in the perpendicular direction (i.e. upwards 'push', Figure 2). The amount of positive limb work done in the perpendicular direction was reduced for the terminating-compared to trailing-limb, which indicates the transfer of bodyweight onto the terminating-limb occurred with a reduced upwards 'push' from the contralateral (trailing) limb. For the trailing-limb, there was also a short period in late stance (following contralateral limb foot contact) when limb power in the parallel direction became positive. This period of positive limb power likely acted to help transfer the body CoM forwards onto the terminatinglimb. No such positive parallel power was evident for the terminating-limb, which would be expected because the CoM was halted on this limb rather than being transferred forwards onto the contralateral limb. There was also positive limb power evident in the mediolateral direction on the trailing-limb during the latter part of the braking phase. This positive power would have occurred because as the CoM was being transferred forward onto the contralateral limb it would have also moved slightly sideways (rightwards) from the trailing (left) limb towards the terminating (right) limb.

The current study has certain limitations. The study only investigated gait terminations that were predictable in terms of where and when they occurred. We chose to focus on such gait terminations because most daily locomotor activity involves volition over where and when to stop, and thus it is important to understand how such terminations are achieved. Future work could determine the mechanical limb work involved in abrupt/unplanned gait terminations and determine whether the contributions from each limb are different to that for predictable/planned gait terminations. The study was also limited by having relatively low participant numbers $(n=8)$ and by focussing on just the two final locomotive-steps involved in terminating gait. Having a participant group of eight is not uncommon for exploratory type studies of human movement/locomotion, and we do not believe that the conclusions made would be any different had we had a bigger group. We focussed on the two final locomotive-steps prior to terminating gait because previous research has shown that terminating gait is accomplished over two walking steps[7-10]. However, we cannot 
rule out the possibility that because the study involved planned gait terminations rather than abrupt terminations, participants didn't begin to slow their forwards velocity during the step preceding the penultimate step (i.e. the step preceding the two steps analysed in the current study). Future work comparing the mechanical limb work involved in abrupt versus planned gait terminations could perhaps explore whether any apparent differences between the two types of gait termination are related to changes occurring in the step preceding the penultimate step. The results presented provide an understanding of the mechanical (external) limb work in all three orthogonal directions as well as the overall limb work, and an understanding of the joints (muscle) work done in achieving such mechanical limb work. However, because only sagittal plane joint work was computed, this may have underestimated the total joint work done. We investigated only sagittal plane joints work because we reasoned that since the task of terminating gait predominantly involves arresting CoM forward velocity, this would mainly be achieved via joint power absorption in the sagittal plane [18]. The relatively small magnitude of mechanical limb power evident in the mediolateral direction indicates that this was indeed the case. Furthermore, frontal and transverse plane joint powers are known to be highly variable [19] and thus their interpretation would likely be problematic. Another limitation was that the two force platforms used to collect GRF data were located next to each other with minimum spacing between them, and thus this may have affected certain participant's stopping strategy more than others, e.g. taller participants may have had to 'chop' their intended foot placements to ensure they were within the bounds of the platforms. However, none of the eight participants were observed to have such difficulties, and all appeared to carry out the stopping task in an apparently natural manner.

In conclusion, this study indicates that during the two locomotor steps of gait termination, the limb that gait is terminated on only does $33 \%$ and $26 \%$ of the overall negative mechanical work done by both limbs on the CoM to terminate gait on the level and declined surface respectively. In other words the trailing-limb does the majority of mechanical work in arresting CoM forwards velocity. Negative joints work was also greater for the trailing-compared to terminating-limb, with negative ankle work in late stance being the foremost contributor. The increased trailing-limb 
422 negative ankle work was associated with an increase in negative limb work in the 423 perpendicular direction, highlighting the ankle's role in slowing rotation of the limb 424 (and thus CoM) over the planted foot (i.e. controlling inverted pendulum). Negative 425 joints work increased on both limbs for declined compared to level surface, 426 particularly so at the knee; indicating kinetic adaptations at the knee are important in 427 controlling the increased CoM lowering required for ramp descent. A peak in 428 negative knee joint power in early stance was associated with a peak in negative 429 limb power in the perpendicular direction, highlighting the knee's role in slowing CoM 430 forwards velocity during weight acceptance onto the limb. These findings may be 431 helpful in designing prosthetic limbs to facilitate walking on ramps.

433 Acknowledgements and funding: Zahraa Abdulhasan is funded by the Higher 434 Committee of Education Development in IRAQ (HCED). 
437

438

[1] Khandoker AH, Lynch K, Karmakar CK, Begg RK, Palaniswami M. Toe clearance and velocity profiles of young and elderly during walking on sloped surfaces. J Neuroeng Rehabil 2010;7(1):1.

[2] Redfern MS, Cham R, Gielo-Perczak K, Gronqvist R, Hirvonen M, Lanshammar $\mathrm{H}$, et al. Biomechanics of slips. Ergonomics 2001;44(13):1138-66.

[3] Cham R, Redfern MS. Changes in gait when anticipating slippery floors. Gait Posture 2002;15(2):159-71.

[4] Donelan JM, Kram R, Kuo AD. Simultaneous positive and negative external mechanical work in human walking. J Biomech 2002;35(1):117-24.

[5] Lay AN, Hass CJ, Richard Nichols T, Gregor RJ. The effects of sloped surfaces on locomotion: an electromyographic analysis. J Biomech 2007;40(6):127685.

[6] Franz JR, Lyddon NE, Kram R. Mechanical work performed by the individual legs during uphill and downhill walking. J Biomech 2012;45(2):257-62.

[7] Jaeger RJ, Vanitchatchavan P. Ground reaction forces during termination of human gait. J Biomech 1992;25(10):1233-6.

[8] Jian Y, Winter DA, Ishac MG, Gilchrist L. Trajectory of the body COG and COP during initiation and termination of gait. Gait Posture 1993;1(1):9-22.

[9] Wearing SC, Urry S, Smeathers JE, Battistutta D. A comparison of gait initiation and termination methods for obtaining plantar foot pressures. Gait Posture 1999;10(3):255-63.

[10] Bishop MD, Brunt D, Pathare N, Patel B. The interaction between leading and trailing limbs during stopping in humans. Neurosci Lett 2002;323(1):1-4.

[11] Cappozzo A, Catani F, Della Croce U, Leardini A. Position and orientation in space of bones during movement: anatomical frame definition and determination. Clin Biomech 1995;10(4):171-8.

[12] Schwartz $\mathrm{MH}$, Rozumalski A. A new method for estimating joint parameters from motion data. J Biomech 2005;38(1):107-16. 
[13] J. Vanrenterghem, D. Gormley, M. Robinson, A. Lees, Solutions for representing the whole-body centre of mass in side cutting manoeuvres based on data that is typically available for lower limb kinematics, Gait Posture 2010;31(4):517-21.

[14] Quanbury AO, Winter DA, Reimer GD. Instantaneous power and power flow in body segments during walking. J Hum Movement Stud 1975;1:59-67.

[15] Lynch J, Robertson DG. Biomechanics of Planned Gait Termination. J Biomech. 2007;40:S500.

[16] Bishop M, Brunt D, Pathare N, Patel B. The effect of velocity on the strategies used during gait termination. Gait Posture. 2004;20(2):134-9.

[17] Kuster M, Sakurai S, Wood GA. Kinematic and kinetic comparison of downhill and level walking. Clin Biomech 1995;10(2):79-84.

[18] Allard P, Lachance R, Aissaoui R, Duhaime M. Simultaneous bilateral 3-D ablebodied gait. Hum Movement Sci 1996;15(3):327-46.

[19] Eng JJ, Winter DA. Kinetic analysis of the lower limbs during walking: What information can be gained from a three-dimensional model? J Biomech 1995;28(6):753-8. 
Figure 1. The 6DoF marker set (front and back view) used to determine body segment motion. Red and green dots - indicate markers used for dynamic tracking (green markers mounted on clusters); blue dots - indicate calibration markers (removed during dynamic tracking).

Figure 2. Group ensemble mean(+/-SD band) limb directional- and total- power profiles $(\mathrm{W} / \mathrm{kg})$ for the trailing- and terminating- limbs. Data are plotted for stance phase of each limb with end of braking-phase indicated by vertical line. Bold line = declined surface; dashed line = level surface.

Figure 3. Group mean (SD) a) negative mechanical (external) limb work (J/kg) in all three orthogonal planes (ML, Parallel and Perpendicular) and b) total limb work for the trailing- and terminating- limbs. Hashed bars = declined surface; solid bars = level surface. The data used to produce this figure has been made available (see supplementary material).

Figure 4. Group ensemble mean (+/-SD band) joint (muscle) power profiles for the hip, knee and ankle joints $(\mathrm{W} / \mathrm{kg})$ of the trailing- and terminating- limbs. Data are plotted for stance phase of each limb with end of braking-phase indicated by vertical line. Bold line = declined surface; dashed line = level surface.

Figure 5. Group mean(SD) negative joint (muscle) work for ankle, knee and hip (A, $504 \mathrm{~K}$, and $\mathrm{H}$ respectively) for the trailing- and terminating- limbs $(\mathrm{J} / \mathrm{kg})$. Hashed bars = declined surface; solid bars = level surface. 


\begin{tabular}{|c|c|}
\hline & Marker location \\
\hline 1 & Headband: Anterior left \\
\hline 2 & Headband: Anterior right \\
\hline 3 & Headband: Posterior left \\
\hline 4 & Headband: Posterior right \\
\hline 5 & Left acromion process \\
\hline 6 & Right acromion process \\
\hline 7 & Jugular notch \\
\hline 8 & Xiphoid process \\
\hline 9 & C7 vertebrae \\
\hline 10 & T8 vertebra on spine \\
\hline 11 & Sacrum cluster: Superior \\
\hline 12 & Sacrum cluster: Left \\
\hline 13 & Sacrum cluster: Right \\
\hline 14 & Sacrum cluster: Inferior \\
\hline 15 & Left iliac crest \\
\hline 16 & Right iliac crest \\
\hline 17 & Left great trochanter \\
\hline 18 & Right great trochanter \\
\hline 19 & Left thigh plate: Proximal anterior \\
\hline 20 & Left thigh plate: Proximal posterior \\
\hline 21 & Left thigh plate: Distal anterior \\
\hline 22 & Left thigh plate: Distal posterior \\
\hline 23 & Left knee: Medial femoral epicondyle \\
\hline 24 & Left knee: Lateral femoral epicondyle \\
\hline 25 & Left shank plate: Proximal anterior \\
\hline 26 & Left shank plate: Distal anterior \\
\hline 27 & Left shank plate: Proximal posterior \\
\hline 28 & Left shank plate: Distal posterior \\
\hline 29 & Left foot: Medial malleolus \\
\hline 30 & Left foot: Lateral malleolus \\
\hline 31 & Left foot: Metatarsal head 1 \\
\hline 32 & Left foot: Metatarsal head 5 \\
\hline 33 & Left foot: Anterior edge \\
\hline 34 & Left foot: Midfoot medial edge \\
\hline 35 & Left foot: Midfoot lateral edge \\
\hline 36 & Left foot: Heel \\
\hline 37 & Right thigh plate: Proximal anterior \\
\hline 38 & Right thigh plate: Proximal posterior \\
\hline 39 & Right thigh plate: Distal anterior \\
\hline 40 & Right thigh plate: Distal posterior \\
\hline 41 & Right knee: Medial femoral epicondyle \\
\hline 42 & Right knee: Lateral femoral epicondyle \\
\hline 43 & Right shank plate: Proximal anterior \\
\hline 44 & Right shank plate: Proximal posterior \\
\hline 45 & Right shank plate: Distal anterior \\
\hline 46 & Right shank plate: Distal posterior \\
\hline 47 & Right foot: Medial malleolus \\
\hline 48 & Right foot: Lateral malleolus \\
\hline 49 & Right foot: Metatarsal head 1 \\
\hline 50 & Right foot: Metatarsal head 5 \\
\hline 51 & Right foot: Anterior edge \\
\hline 52 & Right foot: Midfoot medial edge \\
\hline 53 & Right foot: Midfoot lateral edge \\
\hline 54 & Right foot: Heel \\
\hline
\end{tabular}

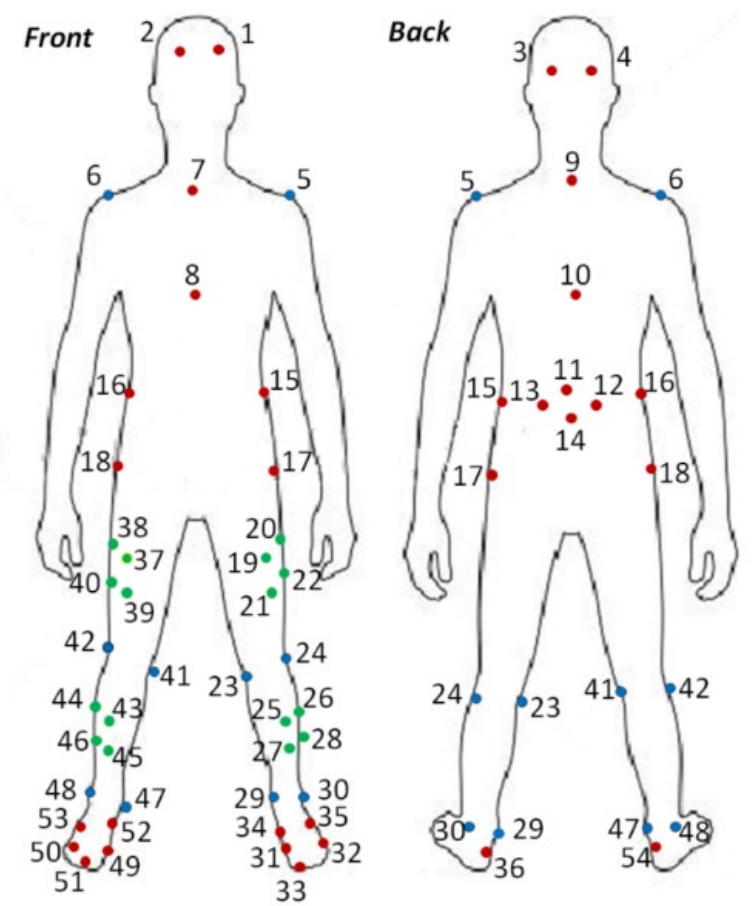

Figure 1. 


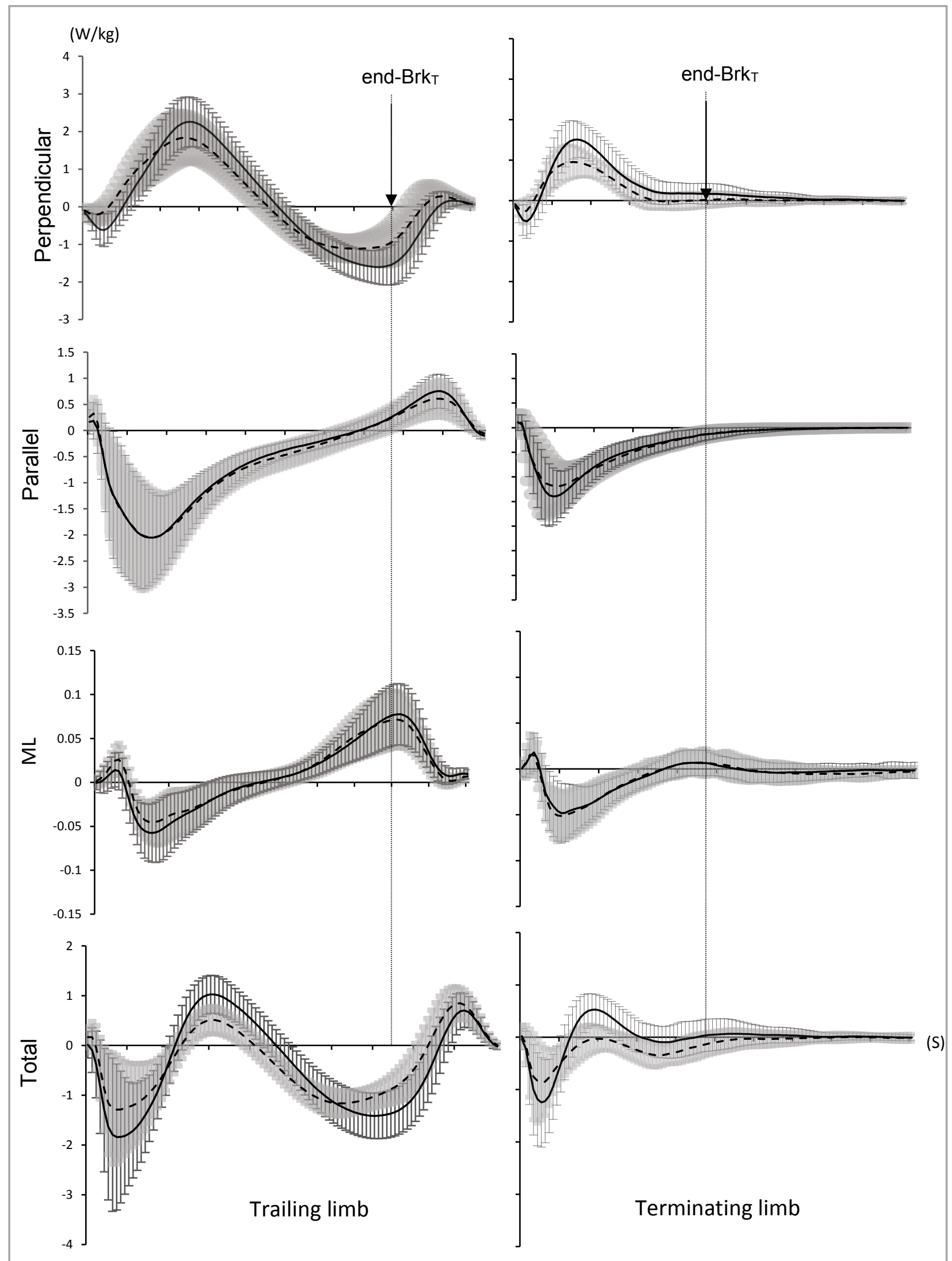

Figure 2 


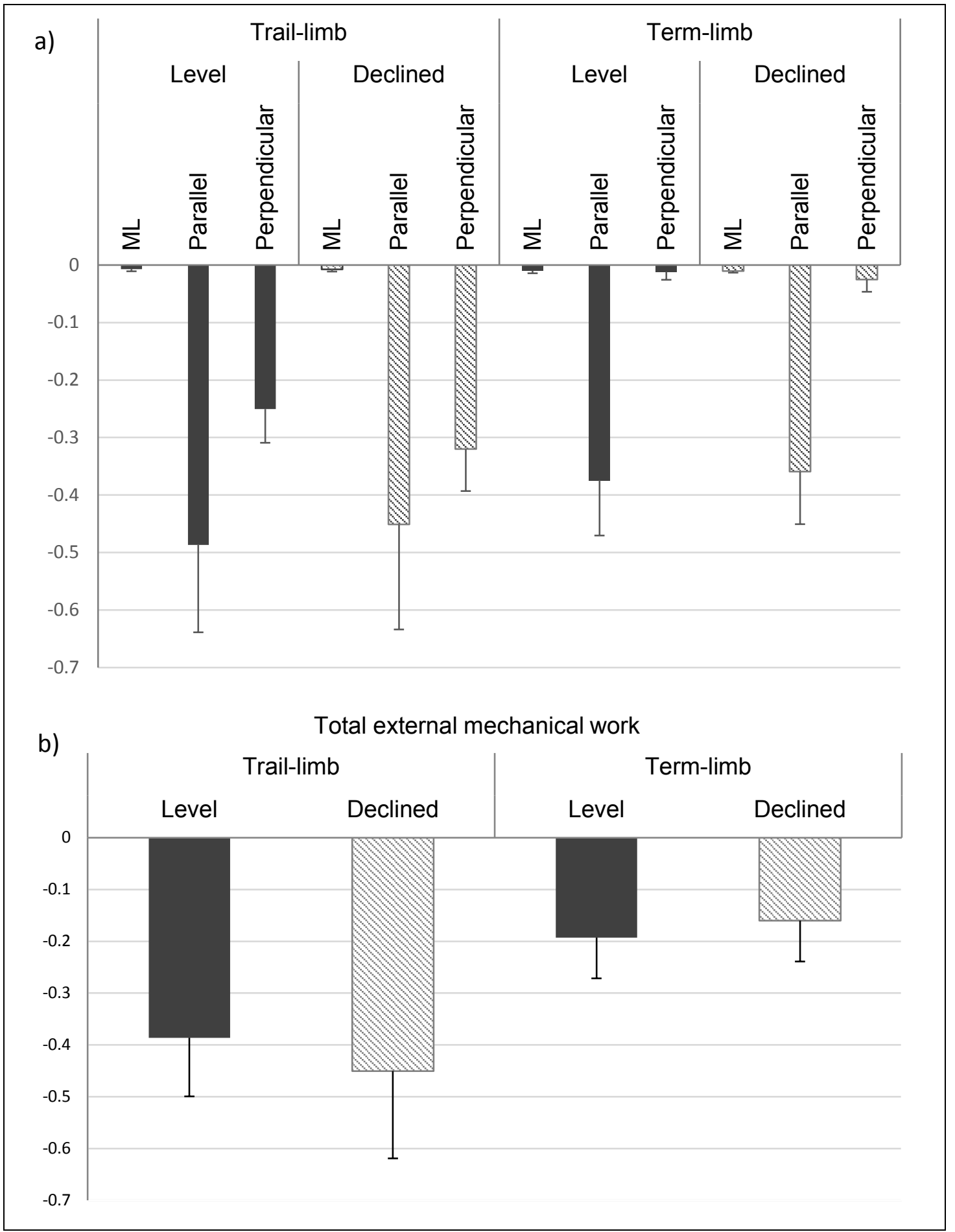

Figure 3 


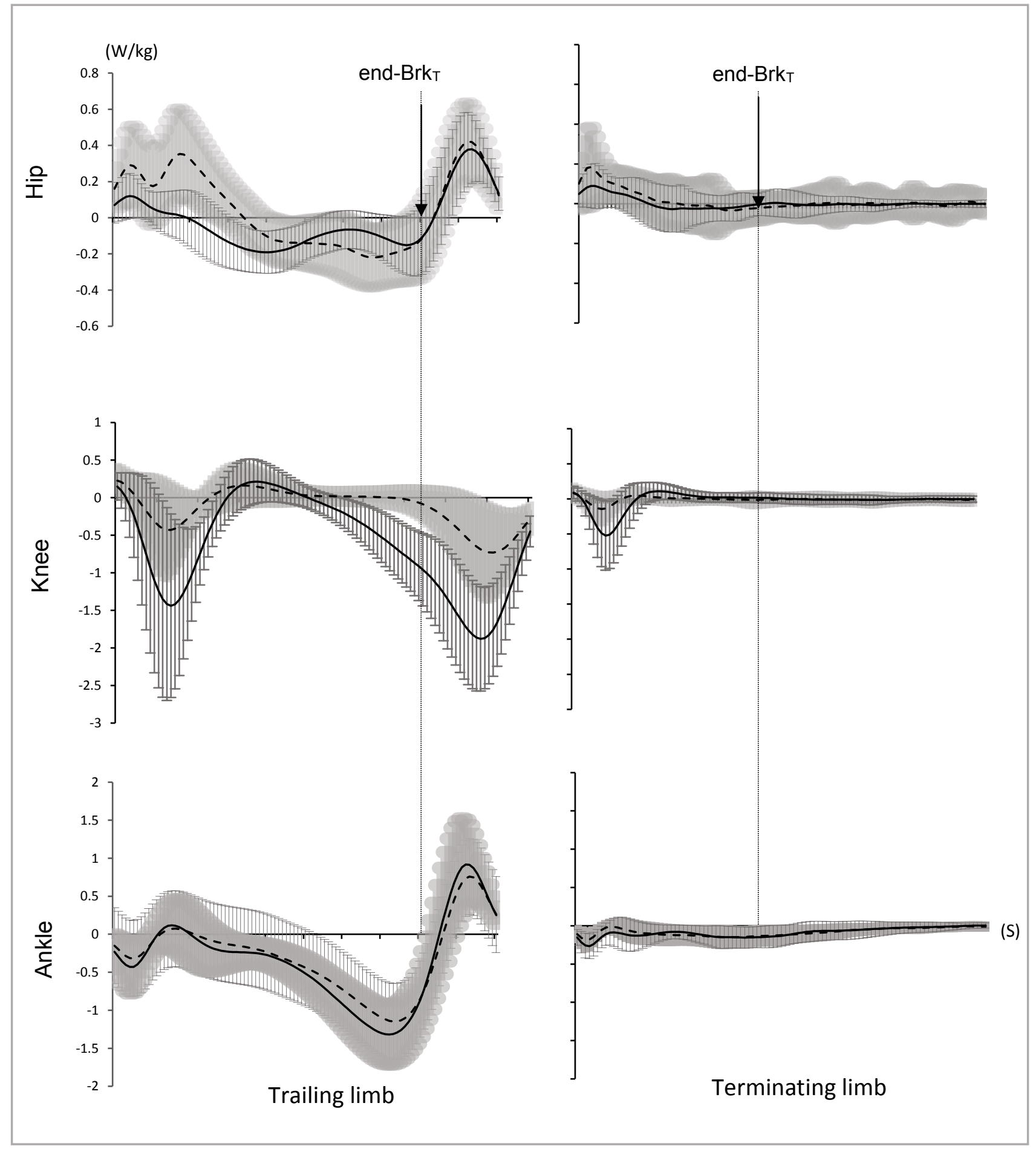

Figure 4 


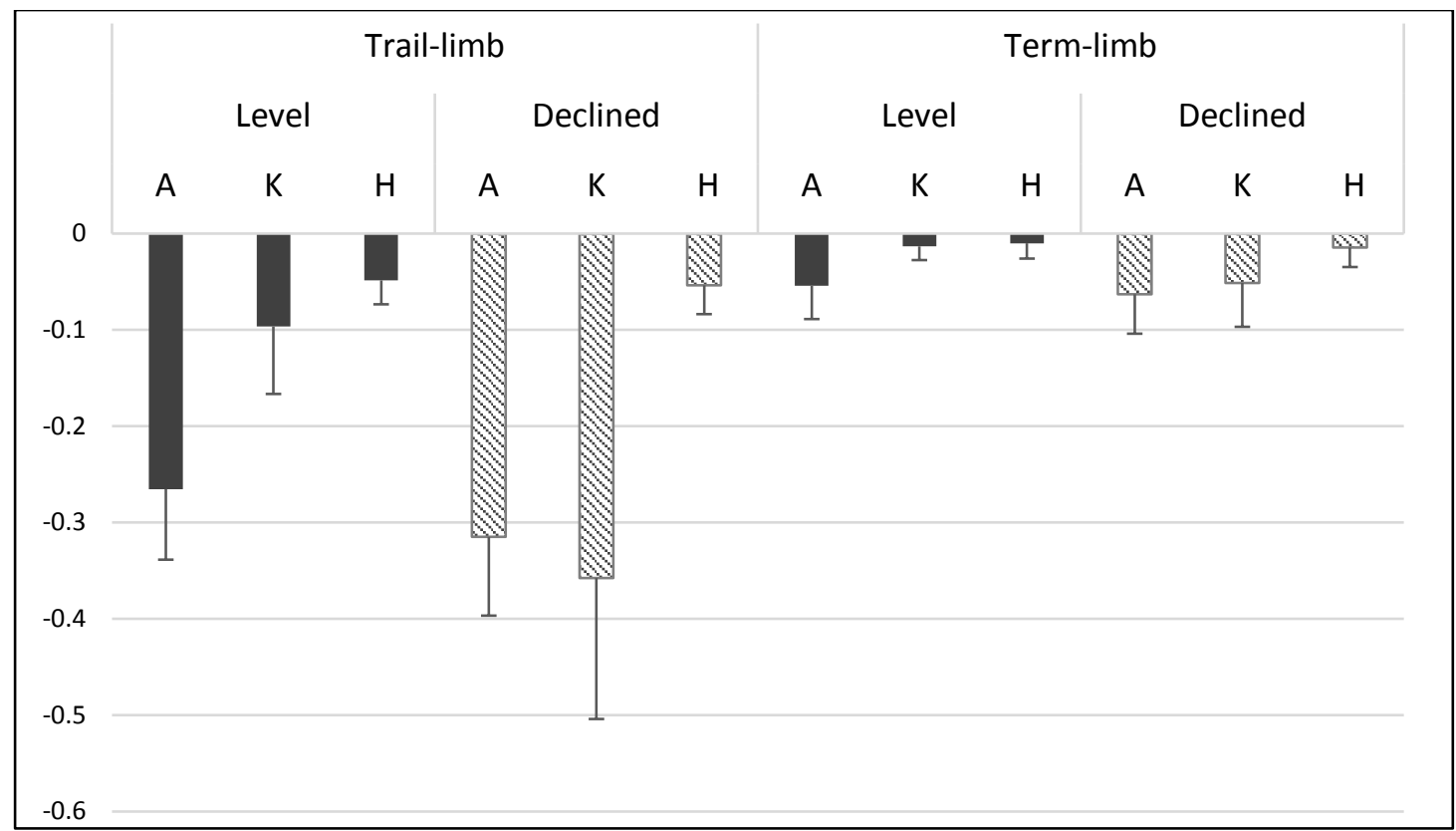

Figure 5 


\section{Supplementary material}

The data contained in the tables below are the data used to produce Figures $3 a$ and $3 b$ in the main article.

Table A. Participant average negative mechanical (external) limb work done (J/kg) in all three orthogonal planes (ML, Parallel and Perpendicular) for both the trailing and terminating limbs, to terminate gait on level and declined surface.

Trailing-limb (J/kg)

Terminating-limb (J/kg)

\begin{tabular}{r|l|l|l|l|l|l|l|l|l|l|l|l}
\hline & \multicolumn{9}{|c}{ Level } & \multicolumn{9}{|l}{ Lecline } & \multicolumn{3}{l|}{ Decline } \\
\hline & ML & Para & Perp & ML & Para & Perp & ML & Para & Perp & ML & Para & Perp \\
\hline$P 1$ & -0.0062 & -0.5686 & -0.1968 & -0.0051 & -0.3763 & -0.2482 & -0.0062 & -0.3416 & -0.0153 & -0.0079 & -0.3830 & -0.0260 \\
\hline$P 2$ & -0.0095 & -0.4547 & -0.2563 & -0.0096 & -0.4200 & -0.3049 & -0.0117 & -0.4224 & -0.0059 & -0.0119 & -0.3613 & -0.0438 \\
\hline$P 3$ & -0.0020 & -0.8243 & -0.3863 & -0.0060 & -0.8714 & -0.4385 & -0.0149 & -0.5487 & -0.0438 & -0.0098 & -0.5193 & -0.0657 \\
\hline$P 4$ & -0.0065 & -0.3976 & -0.2127 & -0.0122 & -0.3002 & -0.2952 & -0.0101 & -0.2969 & -0.0140 & -0.0112 & -0.2156 & -0.0157 \\
\hline$P 5$ & -0.0032 & -0.4372 & -0.2191 & -0.0014 & -0.4592 & -0.2437 & -0.0050 & -0.4592 & -0.0064 & -0.0075 & -0.3145 & -0.0192 \\
\hline$P 6$ & -0.0058 & -0.4683 & -0.2421 & -0.0050 & -0.3181 & -0.2797 & -0.0076 & -0.3393 & -0.0028 & -0.0072 & -0.3207 & -0.0267 \\
\hline$P 7$ & -0.0081 & -0.3340 & -0.2540 & -0.0088 & -0.4991 & -0.4189 & -0.0171 & -0.3332 & -0.0042 & -0.0137 & -0.4404 & -0.0024 \\
\hline$P 8$ & -0.0145 & -0.4078 & -0.2364 & -0.0122 & -0.3636 & -0.3306 & -0.0077 & -0.2535 & -0.0018 & -0.0143 & -0.3185 & -0.0019 \\
\hline Mean & -0.007 & -0.487 & -0.251 & -0.008 & -0.451 & -0.320 & -0.010 & -0.374 & -0.012 & -0.010 & -0.359 & -0.025 \\
\hline$S D$ & 0.004 & 0.152 & 0.059 & 0.004 & 0.183 & 0.073 & 0.004 & 0.096 & 0.014 & 0.003 & 0.091 & 0.021
\end{tabular}

Table B. Participant average total limb work done $(\mathrm{J} / \mathrm{kg})$ for the trailing- and terminating- limbs, to terminate gait on level and declined surface.

Trailing-limb(J/kg) Terminating-limb(J/kg)

\begin{tabular}{r|r|r|r|r|}
\hline participant & Level & Declined & Level & Declined \\
\hline$P 1$ & -0.425 & -0.359 & -0.202 & -0.207 \\
\hline$P 2$ & -0.382 & -0.411 & -0.255 & -0.239 \\
\hline$P 3$ & -0.630 & -0.823 & -0.343 & -0.266 \\
\hline$P 4$ & -0.336 & -0.404 & -0.116 & -0.049 \\
\hline$P 5$ & -0.243 & -0.279 & -0.134 & -0.092 \\
\hline$P 6$ & -0.356 & -0.375 & -0.141 & -0.144 \\
\hline$P 7$ & -0.317 & -0.553 & -0.222 & -0.195 \\
\hline$P 8$ & -0.401 & -0.402 & -0.128 & -0.087 \\
\hline Mean & -0.386 & -0.451 & -0.193 & -0.160 \\
\hline$S D$ & 0.113 & 0.169 & 0.079 & 0.079 \\
\hline
\end{tabular}

